\title{
Electronic density of states derived from thermodynamic critical field curves for underdoped $\mathrm{La}_{2-x} \mathrm{Sr}_{x} \mathrm{CuO} \mathrm{O}_{4+\delta}$
}

\author{
Yung M. Huh and D. K. Finnemore \\ Ames Laboratory, USDOE and Department of Physics and Astronomy, \\ Iowa State University, Ames, IA 50011
}

(November 20, 2018)

\begin{abstract}
Thermodynamic critical field curves have been measured for $\mathrm{La}_{2-x} \mathrm{Sr}_{x} \mathrm{CuO} \mathrm{O}_{4+\delta}$ over the full range of carrier concentrations where superconductivity occurs in order to determine changes in the normal state density of states with carrier concentration. There is a substantial window in the $H-T$ plane where the measurements are possible because the samples are both thermodynamically reversible and the temperature is low enough that vortex fluctuations are not important. In this window, the data fit Hao-Clem rather well, so this model is used to determine $H_{c}$ and $\kappa_{c}$ for each temperature and carrier concentration. Using $N(0)$ and the ratio of the energy gap to transition temperature, $\Delta(0) / k_{B} T_{c}$, as fitting parameters, the $H_{c}$ vs $T$ curves give $\Delta(0) / k_{B} T_{c} \sim 2.0$ over the whole range of $x$. Values of $N(0)$ remain rather constant in the optimum-doped and overdoped regime, but drops quickly toward zero in the underdoped regime. .
\end{abstract}

74.30.Ci., 74.30.Ek, 74.40.+k, 74.60.-w, 74.70.Vy

\section{INTRODUCTION}

In the study of superconductivity in the cuprate materials, the density of states at the Fermi surface, $N(0)$, is a key variable in the determination of the transition temperature. The $\mathrm{La}_{2-x} \mathrm{Sr}_{x} \mathrm{Cu} \mathrm{O}_{4+\delta}$ system is a doped insulator for $x=0$ and the carrier concentration can be increased in an orderly way by holding the oxygep concentration constant and increasing the Sr content. Numerous specific heat measurements 2 目 have been carried out to gain insight into the changes in the density of states. The determination of $N(0)$ from specific heat data, however, is made difficult because the lattice specific heat is large and because the upper critical field is above the reach of the magnets available. Another approach to the changes in $N(0)$ is through the study of optical conductivity. ${ }^{-}$By assuming a Drude like absorption and integrating over all frequencies, it was found that $N(0)$ rises in the underdoped region and levels off in the overdoped region.

The purpose of this work is to measure the thermodynamic critical field curves, $H_{c}$ vs. $T$, for the $\mathrm{La}_{2-x} \mathrm{Sr}_{x} \mathrm{CuO} \mathrm{O}_{4+\delta}$ system over the entire range of superconductivity. Thermodynamic critical field data are important in their own right because they give the free energy difference between the superconducting and normal state and a good starting place for theory. In addition, we wish to compare the thermodynamic critical fields of $L a_{2-x} S r_{x} C u O_{4+\delta}$ with those of classical superconductors and with the Bardeen-Cooper-Schrieffer (BCS) picture. Within the BCS model and succeeding strong coupling modifications, 6 the ratio of the critical field at $T=0$ to the critical temperature, $H_{o} / k_{B} T_{c}$ is related to $N(0)$ and the curvature of the $H_{c} v s . T$ curve is related to the ratio of the energy gap at $T=0$ to transition temperature, $\Delta(0) / k_{B} T_{c}$. The goal is to measure these changes in $N(0)$ and $\Delta(0) / k_{B} T_{c}$ as the $S r$ content changes.

There is a vocabulary problem in the discussion of these data in that the thermodynamic critical field curve and the free energy difference are most closely related to the condensation energy per Cooper pair rather than the energy gap. In BCS, the two quantities are equivalent, and we have used the terms interchangeably in the discussion here because we are comparing the results to BCS.

A preliminary single crystal study recently published illustrates the success and difficulties of measuring thermodynamic critical fields in this system. Extensive measurements] of a high quality single crystal of $L a_{1.90} \mathrm{Sr}_{0.10} \mathrm{CuO} \mathrm{O}_{4+\delta}$ have shown that the superconducting magnetization curves, $M_{s c} v s$. $H$, of this moderately underdoped high temperature superconductor obeys the Hao-Clem model over a wide range of temperature, $T$, and magnetic fields, $H$. The Hao-Clem model is a variational calculation developed originally for the $\mathrm{YBa}_{2} \mathrm{Cu}_{3} \mathrm{O}_{7-\delta}$ system and it was shown to describe the magnetization curves, well for both $\mathrm{YBa}_{2} \mathrm{Cu}_{3} \mathrm{O}_{7-\delta} \mathrm{Q}$ and $\mathrm{La}_{2-x} \mathrm{Sr}_{x} \mathrm{CuO} \mathrm{O}_{4+\delta}$ system. In the $\mathrm{La}_{1.90} \mathrm{Sr}_{0.10} \mathrm{CuO} \mathrm{O}_{4+\delta}$ studies, it also was found that the normal state background magnetization follows a 2D Heisenberg model 10 for the $\mathrm{Cu}$ spins with values close to those measured previously by Nakano and coworkers. 11 In addition, this $x=0.10$ sample shoyed a vortex fluctuation contribution to the magnetization 12 close to $T_{c}$. The presence of these fluctuations limits the temperature range of applicability of Hao-Clem because they introduce a new term in the free energy. We only use Hao-Clem in regions where these fluctuations do not contribute significantly. At low temperature irreversibility of the magnetization prevents equilibrium magnetization measurements and close to $T_{c}$, fluctuations make Hao-Clem inapplicable. Even so, there is a window for each $\mathrm{x}$-value where Hao-Clem works well and thermodynamic critical fields can be measured. 


\section{EXPERIMENT}

Both single crystals and grain-aligned-powders are used in this work. In the early part of the experiment, it was thought that it would be easier to control the oxygen content in grain-aligned-powders than in single crystals because the diffusion distance for oxygen would be less. Single crystals from three different sources, however, have given essentially the same result as the powders. Magnetically aligned samples were prepared by grinding appropriate amounts of $\mathrm{La}_{2} \mathrm{O}_{3}, \mathrm{SrCO}_{3}$ and $\mathrm{CuO}$ in an agate mortar and pestle. Mixed and ground powders were pressed into pellets, placed in an alumina boat, and initially fired for $24 \mathrm{~h}$ at $750^{\circ} \mathrm{C}$. This was followed by grinding, pelletizing, and firing several times at $850^{\circ} \mathrm{C}$ and $970^{\circ} \mathrm{C}$ for 48 and $72 h$ respectively. After several cycles at these temperatures, the grinding, pelletizing and sintering was done at successively higher temperatures of $1000^{\circ} \mathrm{C}, 1050^{\circ} \mathrm{C}$, and $1100^{\circ} \mathrm{C}$ in a tube with oxygen flowing at $2.5 \mathrm{~cm}^{3} / \mathrm{min}$ for $24 \mathrm{~h}$ each time. Measurements of $T_{c}$ and zero-field-cooled Meissner screening fraction at $1.0 \mathrm{mT}$ were carried out as the first test for sample quality. The final pellet was ground to a particle size of about $20 \mu \mathrm{m}$, mixed with a low viscosity epoxy (Epotek 301), oriented in a magnetic field of $8.0 \mathrm{~T}$, and then the epoxy was allowed to harden. X-ray diffraction patterns showed only $(00 \ell)$ peaks. The full width at half maximum of the (008) peak was $5^{\circ}$. Inductively coupled plasma (ICP) measurements were used to determine the $S r$ content or $\mathrm{x}$-value.

Magnetization data were taken with $H \| c$ in a Quantum Designs magnetometer with a $3-\mathrm{cm}$ scan for the $x=0.13$ single crystal and a $6-\mathrm{cm}$ scan for all others. In this magnetometer, the sample is moved through a series of three pick-up coils, and the length of scan determines the uniformity of magnetic field seen by the sample during the scan. We selected the sample that has the highest $T_{c}$ for any given x-value and this corresponded well with literature values in all cases. In the region of $x=1 / 8$, the superconducting transition widths are about double the width away from this region. In this work, the magnetization is taken to be thermodynamically reversible if the difference in the field increasing and field decreasing magnetization is less than one percent of the average magnetization. The range of temperatures was $1.4 \mathrm{~K}$ to $300 K$, and the range of magnetic fields was 0 to $7 T$.

\section{RESULTS AND DISCUSSION}

The superconducting transition temperature, $T_{c}$, for both the single crystals (solid triangles) and the grain aligned powders (solid circles) are rather close to the literature values as shown in Fig. 1. There is a very clear dip in the curve near $x=1 / 8$ similar to that found by others and in $L a_{2-x} B a_{x} C u O_{4+\delta} .13$ The $T_{c}$ values for the grain aligned samples agree well with the single crystals published here as well as those reported in the literature.14

\section{A. Normal State Magnetization}

There is a very predictable and regular background magnetization, $M_{b}$, that appears to arise primarily from the $\mathrm{Cu}$ spin system. Measurements of $M_{b}$ in the normal state were made between $60 K$ and $200 K$ and found to fit the relation,

$$
M_{b}=C H+M_{s} \tanh (\beta H),
$$

where the dominant term is the linear $C H$ term. In Eq.1, $C, M_{s}$, and $\beta$ are fit constants. Figure 2 shows that the magnitude of this magnetization is on the order of $10^{-5}$ to $10^{-3} \mu_{B} / C u$ atom. As reported earlier for the $x=0.10$ single crystal, the $M_{s} \tanh (\beta H)$ term saturates at about $0.1 T$ and the value of $M_{s}$ ranges between $3-6 \times$ $10^{-5} \mu_{B} / C u$ atom. The $M_{s} \tanh (\beta H)$ term is small and both $M_{s}$ and $\beta$ are nearly independent of temperature and $\mathrm{x}$-value in the range measured. The value of the slope of the linear term, C, is rather close to the values reported by Nakano and coworkers 11 as shown by Fig. 3. The solid lines are from Ref. 5 for $x=0.10,0.14,0.16,0.20,0.26$ running from bottom to top. To determine the normal state magnetization at temperatures below $T_{c}$, the value of $C$ is linearly extrapolated from the $60 K$ to $200 K$ data to the desired temperature and $M_{b}$ is determined from Eq. 1. Because the constants, $M_{s}$ and $\beta$ do not change with temperature, they are taken to be the average of the values determined from $60-200 \mathrm{~K}$.

\section{B. Irreversibility field}

In the over-doped region, the irreversibility field, $H_{i r r}$, has essentially the same values as the optimum doped sample as shown in the reduced temperature, $T / T_{c}$ plot of Fig. 4. In the underdoped region, the flux pinning is much weaker and $H_{i r r}$ drops to lower values. As Janossy and coworkers 15 showed for $\mathrm{YBa}_{2} \mathrm{Cu}_{3} \mathrm{O}_{7-\delta}$, as charge carriers are removed from the cuprate superconductor, the material becomes more anisotropic and the pinning is reduced.

\section{Thermodynamic critical field curves}

To determine the magnetization of the superconducting charge carriers, $M_{s c}$, the background magnetization is subtracted from the total measured magnetization, $M_{t}$, by, $M_{s c}=M_{t}-M_{b}$. To illustrate the results, Fig. 5 shows superconducting magnetization data as a function of magnetic field for the interval between $H_{i r r}$ and the field where vortex fluctuations become important. In the 
first attempt to fit these data to the Hao-Clem model, both $H_{c}$ and $\kappa_{c}$ are used as adjustable parameters. Here, $\kappa_{c}=H_{c 2} / \sqrt{2} H_{c}$ is approximately the ratio of the penetration depth to the coherence distance. Usually the data do not extend very close to $H_{c 2}$, so the fit is not very sensitive to $\kappa_{c}$. Hence, the average of $\kappa_{c}$ for any given $x$-value is used and $H_{c}$ is adjusted slightly for the best fit. For the optimum-doped and overdoped range, $\kappa_{c}$ ranges from 80 to 120 . The maximum value of $\kappa_{c}$ is about 200 for $x=0.10$ and it falls to about 120 again in the $x$ equal to $0.07-0.08$ range. The fits to Hao-Clem and the resulting $H_{c} v s . T$ curve are shown in Fig.6.

The critical field curve data for samples in the overdoped regime are shown in Fig. 7a, and the data for the underdoped regime are shown in Fig. 7b. The solid lines through the data are best fit parabolas of the form $H_{c}=H_{o}\left[1-\left(T / T_{c}\right)^{2}\right]$. In the overdoped data of Fig. 7a, the ratio of $H_{o} / T_{c}$ remains relatively constant, and in the underdoped regime, the ratio of $H_{o} / T_{c}$ drops quickly as the $\mathrm{x}$-value or charge carrier concentration is reduced.

\section{Comparison of data with BCS theory}

The BCS theory has been very successful describing the thermodynamic critical field curves of classical superconductors. Within the theory, the $H_{c}$ value at any given temperature can be calculated from two microscopic variables, the ratio of the energy gap to the thermal energy, $\Delta(T) / k_{B} T$ at that temperature and the value of the density of states, $N(0)$. Modifications of BCS to include strong-coupling effects 6 show that the ratio of the energy gap at $T=0$ to $k_{B} T_{c}, \Delta(0) / k_{B} T_{c}$ rises above the weakcoupling BCS value of $\Delta(0) / k_{B} T_{c}=1.76$ to values above $\Delta(0) / k_{B} T_{c}=2$ for strong-coupling superconductors like $\mathrm{Pb}$ and $\mathrm{Hg}$. For these materials, the temperature dependence of $\Delta(T) / \Delta(0)$ has approximately the same shape as the original BCS form.

To compare and contrast these $\mathrm{La}_{2-x} \mathrm{Sr}_{x} \mathrm{CuO} \mathrm{O}_{4+\delta}$ superconductors with classical superconductors, we assume that strong-coupling BCS theory applies to both classes of material and do a two parameter fit of the thermodynamic critical field curves using $N(0)$ and $\Delta(0) / k_{B} T_{c}$ as fitting variables. In this fitting, $N(0)$ controls the magnitude of $H_{c}$ and $\Delta(0) / k_{B} T_{c}$ controls the curvature of the $H_{c} v s . T$ plot. The results are shown in Fig. 8a and $8 \mathrm{~b}$. The value of $\Delta(0) / k_{B} T_{c}$ is consistently in the 1.9 to 2.1 range with the underdoped samples being very similar to the overdoped samples. The specific heat $\gamma$, which is related to $N(0)$ by $\gamma=2 / 3 \pi^{2} k_{B}^{2} N(0)$, is relatively constant at $3.0 \mathrm{~mJ} / \mathrm{mol} K^{2}$ in the optimum and overdoped region. The value of $\gamma$ then drops quickly to a minimum at the $x=1 / 8$ region, rises to a maximum at $x=0.010$, and falls quickly as $x$ falls in the underdoped regime. Measured values of $T_{c}$ are also shown in Fig. $8 \mathrm{~b}$ for comparison with the trajectory of $\gamma v s$. $x$. A much more detailed discussion of the data and the analysis is given elsewhere.16

\section{CONCLUSIONS}

There is a reasonable window of thermodynamic reversibility where equilibrium magnetization curves can be measured in the $\mathrm{La}_{2-x} \mathrm{Sr}_{x} \mathrm{CuO}_{4+\delta}$ system. The superconducting magnetization curves obey Hao-Clem rather well, even in the underdoped region as long as data are taken in the range of applicability of the model. As the xvalue drops, $H_{i r r}$ drops giving a wider region of the $H-T$ plane. The range of vortex fluctuations, however, also increases so that the temperature interval where Hao-Clem can be used remains roughly constant over the x-values measured.

Thermodynamic critical field curves for $L a_{2-x} \mathrm{Sr}_{x} \mathrm{CuO}_{4+\delta}$ are rather similar to those of classical superconductors. From the curvature of the $H_{c} v s . T$ plots, one can derive a $\Delta(0) / k_{B} T_{c}$ value that is roughly independent of $x$ and equal to about 2.0. From the magnitude of the $H_{c} v s . T$ plot, the specific heat $\gamma$ remains nearly constant at $3.0 \mathrm{~mJ} / \mathrm{mol} \mathrm{K}^{2}$ in the overdoped region. In the underdoped region, $\gamma$ falls to a minimum at the $x=1 / 8$ value, rises to a maximum at $x=0.10$, and then falls quickly toward zero as $x$ approaches 0.06 . In the broad picture, $N(0)$ is roughly constant in the overdoped regime, and $N(0)$ falls quickly in the underdoped regime. In this broad feature, the data are similar to the change in the density of states found by integrating optical absorption data over all frequencies.4

\section{ACKNOWLEDGMENTS}

Ames Laboratory is operated for the U. S. Department of Energy by Iowa State University under contract No. W-7405-ENG-82 and supported by the DOE, the Office of Basic Energy Sciences.

${ }^{1}$ T. Timusk and B. Statt. The pseudogap in high temperature superconductors: an experimental overview, Rep. Prog. Phys. 62, 61 (1999).

${ }^{2}$ N. E. Phillips, R. A. Fisher, A. Schilling, B. Bufferteau, T. E. Hargreaves, C. Marcenat, R. Calemczuk, A. S. O'Connor, K. W. Dennis, and R. W. McCallum, Physica B259-261, 546 (1999).

${ }^{3}$ J. W. Loram, K. A. Mirza, J. R. Cooper, and J. L. Tallon, J. Phys. Chem. Solids, 59, 2091 (1998).

${ }^{4}$ K. Yamada, C. H. Lee, K. Kurahashi, J. Wada, S. Wakimoto, S. Ueki, H. Kimura, Y. Endoh, S. Hosoya, G. Shirane, R. J. Birgeneau, M. Greven, M. A. Kastner, and Y. J. Kim, Phys. Rev. B 57, 6165 (1998). 
${ }^{5}$ J. Bardeen, L. N. Cooper, and J. R. Schrieffer, Phys. Rev. 108, 1175 (1957).

${ }^{6}$ J. C. Swihart, D. J. Scalapino, and Y. Wada, Phys. Rev. Lett. 14, 106 (1965).

${ }^{7}$ Y. M. Huh, J. E. Ostenson, F. Borsa, V. G. Kogan, D. K. Finnemore, A. Vietkin, A. Revcolevschi, and M. -H. Julien, Phys. Rev. B63, 64512 (2001).

${ }^{8}$ Z. Hao and J. R. Clem, Phys. Rev. Lett. 67, 2371 (1991).

${ }^{9}$ Z. Hao, J. R. Clem, M. W. McElfresh, L. Civale, A. P. Malozemoff, and F. Holzberg, Phys. Rev. B43, 2844 (1991).

${ }^{10}$ L. J. de Jongh, Magnetism and Magnetic Materials Ed. C. D. Graham and J. Rhyne, AIP Conference Proceedings No. 10 (American Institute of Physics, New York,1973 p. 561)

11 T. Nakano, M. Oda, C. Manabe, N. Momona, Y. Miura, and M. Ido, Phys. Rev. B40,16000 (1994).

${ }^{12}$ L. N. Bulaevskii, M. Ledvij, and V. G. Kogan, Phys. Rev. Lett. 68, 3773 (1992), P. H. Kes, C. J. van der Beek, M. P. Maley, M. E. McHenry, D. A. Huse, M. J. Menken, and A. A. Minovsky, Phys. Rev. B67, 2383 (1991).

${ }^{13}$ Y. Zhu, A. R. Moodenbaugh, X. Z. Cai, J. Tafto, M. Suenaga, D. O. Welch, Phys. Rev. Lett. 73, 3026 (1994).

${ }^{14}$ P. G. Radaelli, D. G. Hinks, A. W. Mitchell, B. A. Hunter, J. L. Wagner, B. Dabrowski, K. G. Vandervoort, H. K. Viswanathan, and J. D. Jorgensen, Phys. Rev. B 49, 4163 (1994).

15 B. Janossy, D. Prost, S. Pekker, and L Fruchter, Physica C 181, 51 (1991.

${ }^{16}$ Yung M. Huh, Ph.D. thesis, Iowa State University, 2001.

FIG. 1. $T_{c}$ values for both single crystals (solid triangles) and grain aligned powders (solid circles)

. The solid line is a sketch from data of Ref. 14.

FIG. 2. Normal state magnetization at $80 K$ for the full range of x-values. The solid lines are fits of the data to Eq.1. The inset expands the low field portion.

FIG. 3. Comparison of the slope of the normal state magnetization with the values obtained by Ref. 5 . Bottom to top, the solid lines for Ref. 5 run from $x=0.10,0.14,0.16,0.20,0.26$

FIG. 4. Irreversibility curves.

FIG. 5. $M_{s c} v s . H$ for $x=0.156$ grain aligned sample.

FIG. 6. A fit of the grain aligned $x=0.156$ data to the universal Hao-Clem curve for $\kappa_{c}=91$. The inset shows the value of $H_{c}$ used to fit the curves.

FIG. 7. Thermodynamic critical field curves in both a) overdoped region, and b)optimum doped and underdoped regions.
FIG. 8. Results of a two parameter fit to $H_{c}$ vs. $T$ data, a) $\Delta(0) / k_{B} T_{c}$, and b) specific heat $\gamma$. 


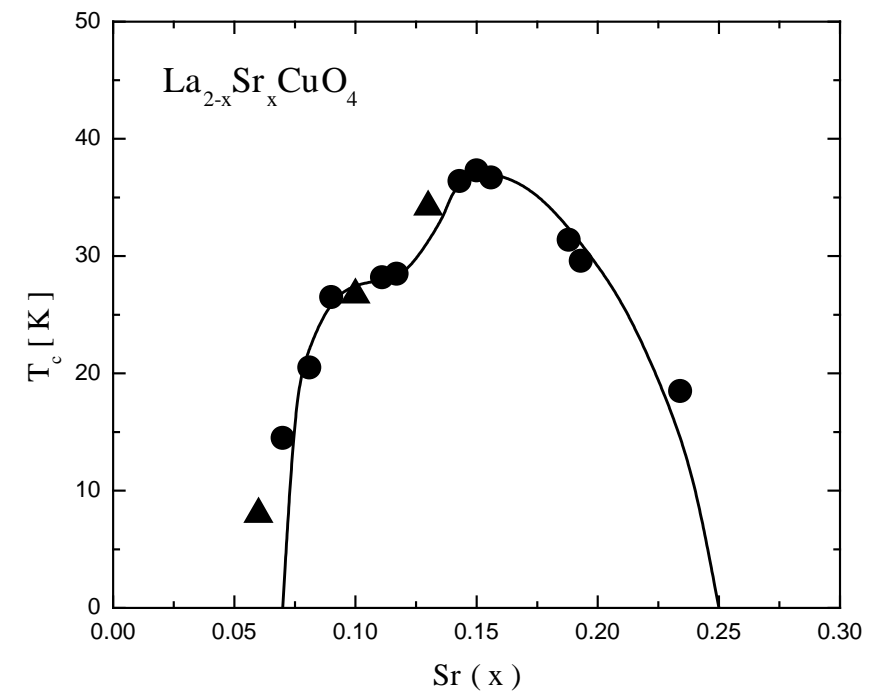

FIG. 1

Yung M. Huh and D. K. Finnemore 


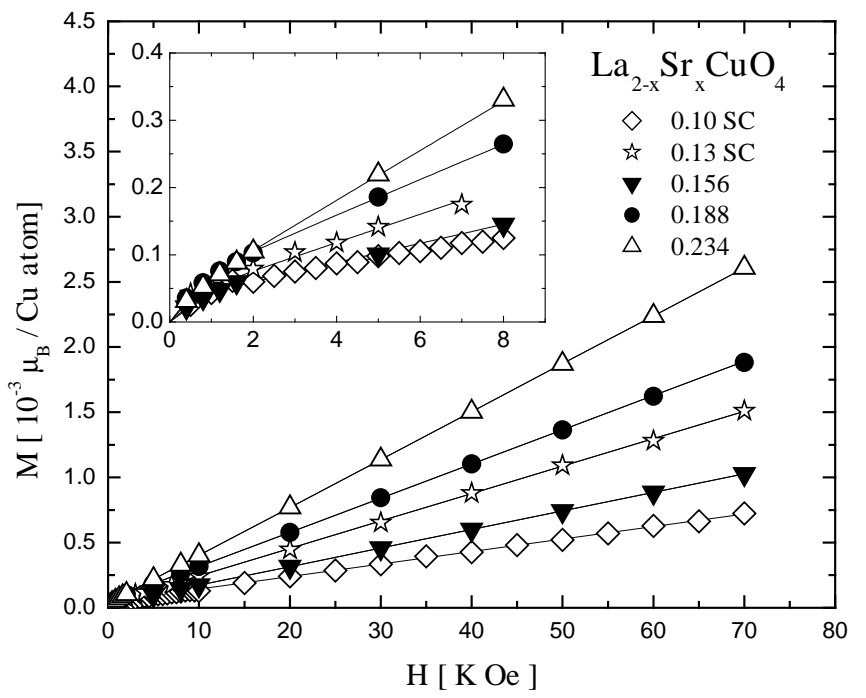

FIG. 2

Yung M. Huh and D. K. Finnemore 


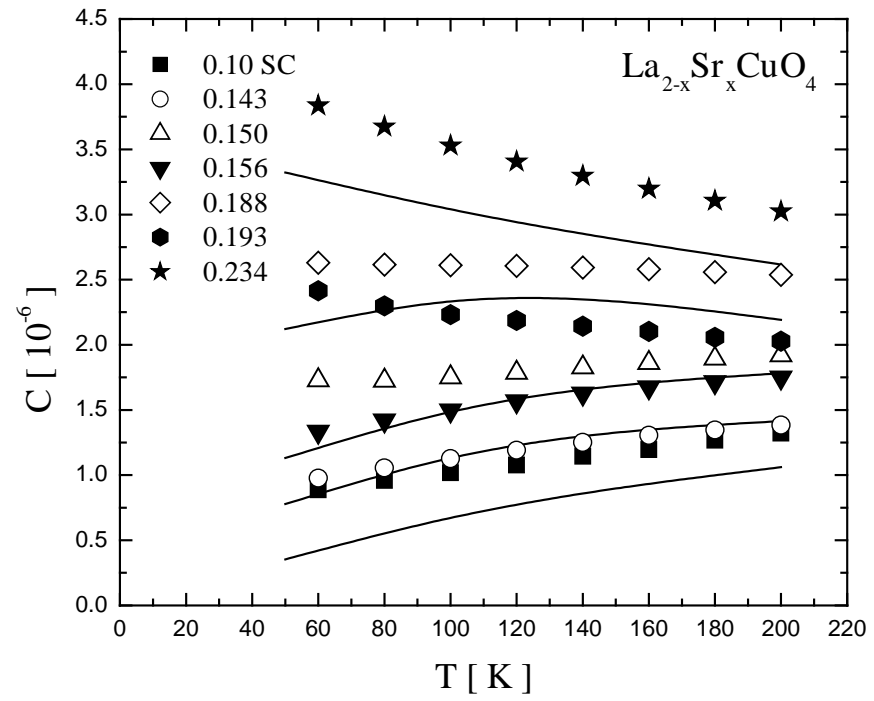

FIG. 3

Yung M. Huh and D. K. Finnemore 


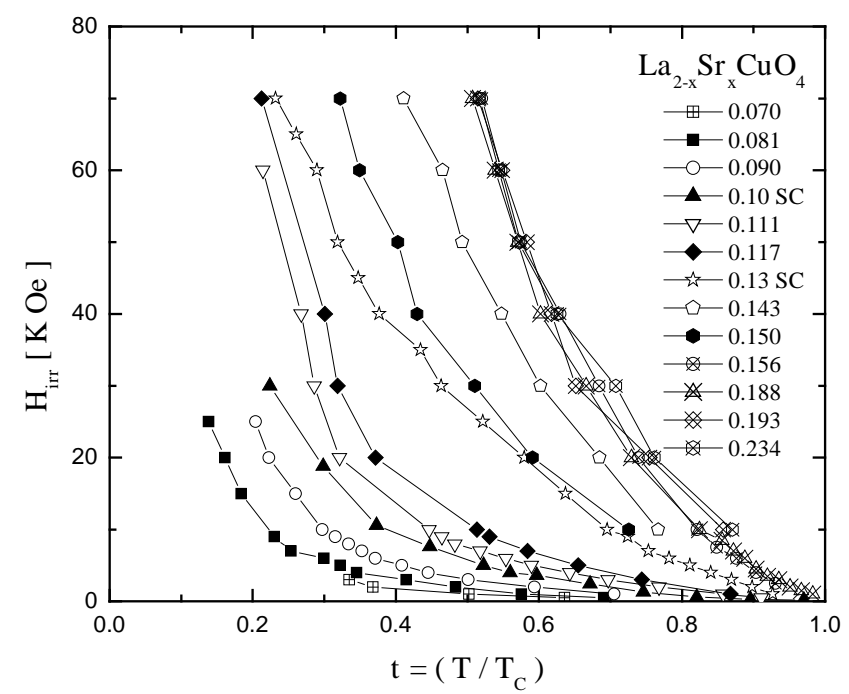

FIG. 4 


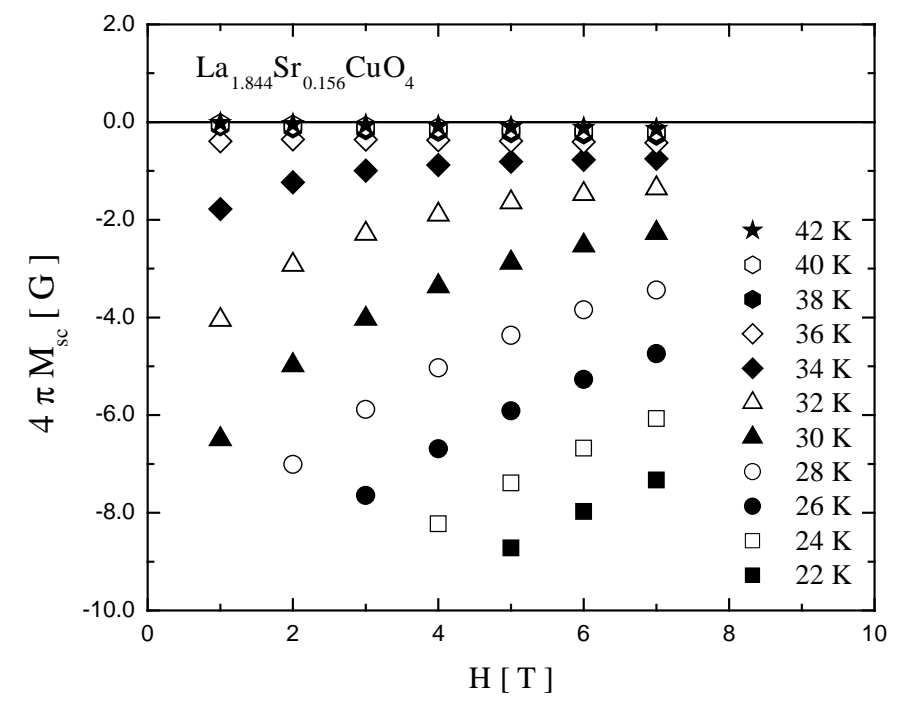

FIG. 5 


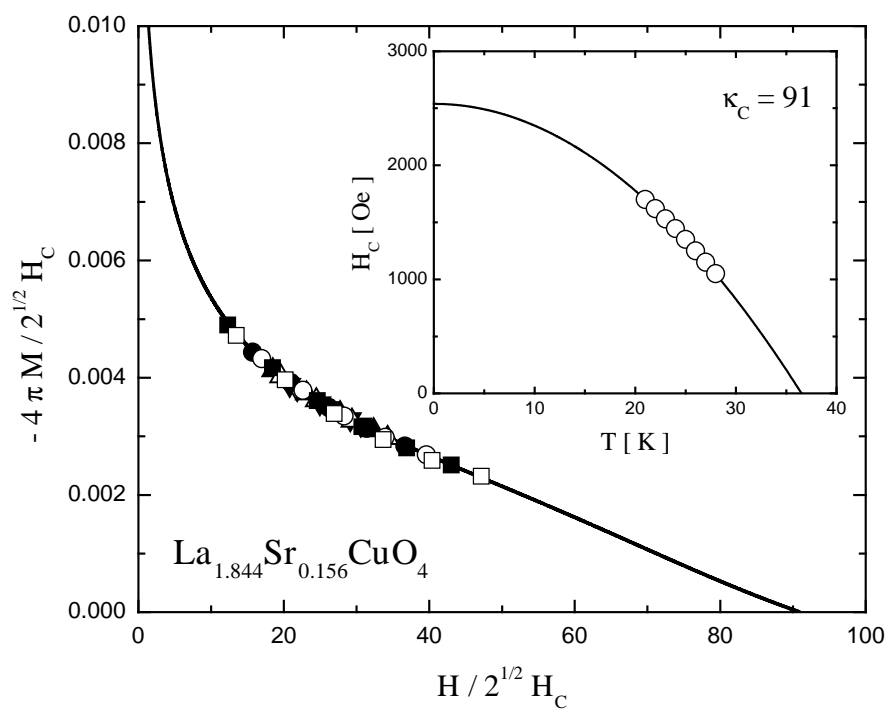

FIG. 6

Yung M. Huh and D. K. Finnemore 


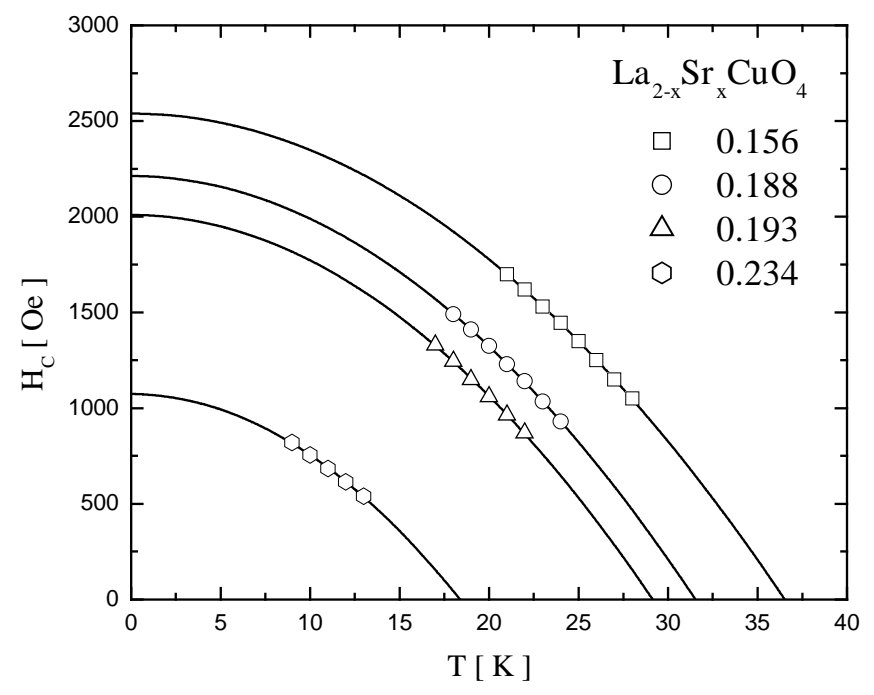

FIG. 7a

Yung M. Huh and D. K. Finnemore 


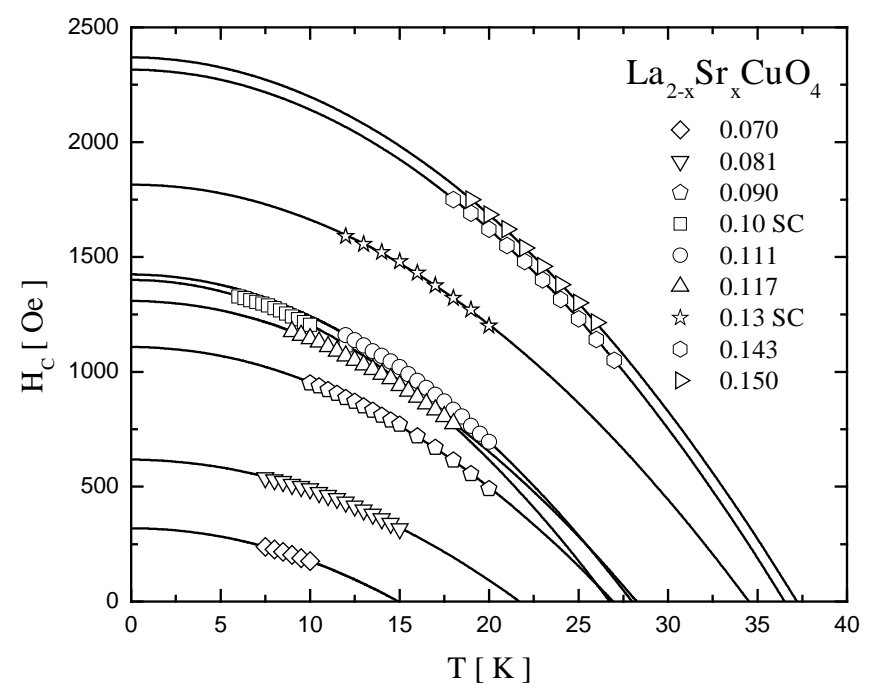

FIG. 7b

Yung M. Huh and D. K. Finnemore 


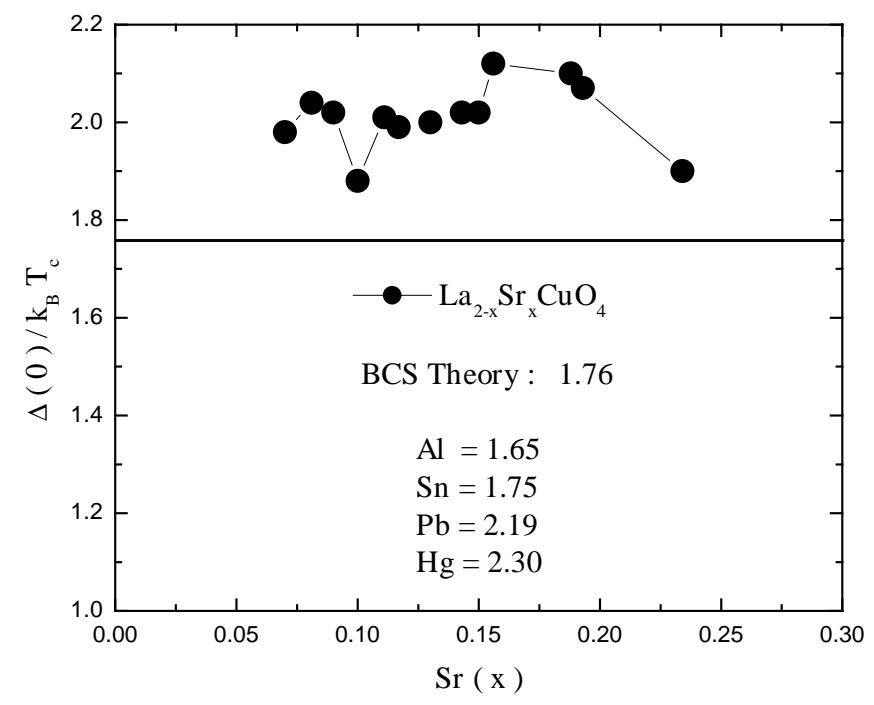

FIG. 8a

Yung M. Huh and D. K. Finnemore 


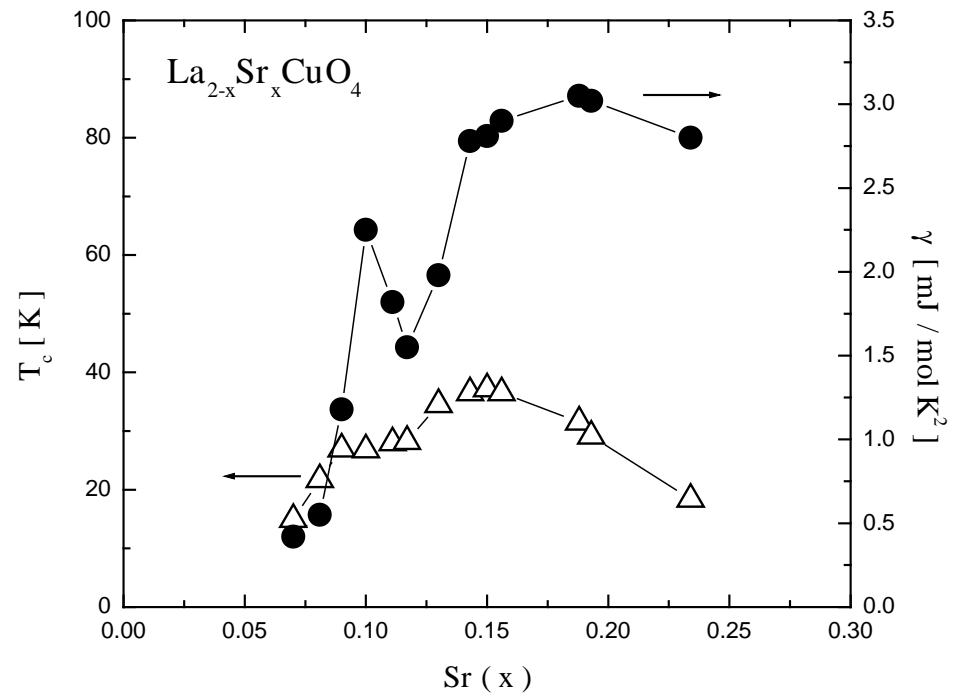

FIG. 8b

Yung M. Huh and D. K. Finnemore 